\title{
Assessment of joystick and wrist control in hand-held articulated laparoscopic prototypes
}

\author{
Linde M. Okken • Magdalena K. Chmarra • \\ Ellen Hiemstra • Frank Willem Jansen • \\ Jenny Dankelman
}

Received: 4 March 2011 / Accepted: 15 December 2011/Published online: 11 January 2012

(C) The Author(s) 2012. This article is published with open access at Springerlink.com

\begin{abstract}
Background Various steerable instruments with flexible distal tip have been developed for laparoscopic surgery. The problem of steering such instruments, however, remains a challenge, because no study investigated which control method is the most suitable. This study was designed to examine whether thumb (joystick) or wrist control method is designated for prototypes of steerable instruments by means of motion analysis.

Methods Five experts and 12 novices participated. Each participant performed a needle-driving task in three directions (right $\rightarrow$ left, up $\rightarrow$ down, and down $\rightarrow$ up) with two prototypes (wrist and thumb) and a conventional instrument. Novices performed the tasks in three sessions, whereas experts performed one session only. The order of performing the tasks was determined by Latin squares design. Assessment of performance was done by means of five motion analysis parameters, a newly developed matrix for assigning penalty points, and a questionnaire.

Results The thumb-controlled prototype outperformed the wrist-controlled prototype. Comparison of the results obtained in each task showed that regarding penalty points, the up $\rightarrow$ down task was the most difficult to perform.

Conclusions The thumb control is more suitable for steerable instruments than the wrist control. To avoid
\end{abstract}

L. M. Okken · E. Hiemstra · F. W. Jansen

Department of Gynecology, Leiden University Medical Center, Leiden, The Netherlands

M. K. Chmarra $(\square)$ · F. W. Jansen · J. Dankelman Department of BioMechanical Engineering, Delft University of Technology, Mekelweg 2, 2628 CD Delft, The Netherlands e-mail: m.k.chmarra@tudelft.nl uncontrolled movements and difficulties with applying forces to the tissue while keeping the tip of the instrument at the constant angle, adding a "locking" feature is necessary. It is advisable not to perform the needle driving task in the up $\rightarrow$ down direction.

Keywords Minimally invasive surgery $\cdot$ Hand-held articulated instruments $\cdot$ Motion analysis $\cdot$ Needle-driving

One of the main difficulties of laparoscopic surgery is that it is performed via an incision point, which acts as a pivoting point [1]. As a result, the number of degrees of freedom (DOFs) is limited from six to four [1, 2]. This means that movements in laparoscopy are restricted. By consequence, it often is difficult to approach anatomic structures from some directions with conventional laparoscopic instruments. Moreover, it is difficult to perform technically demanding maneuvers, e.g., intracorporeal suturing and knot tying [3,4]. To overcome the restriction in movements, various steerable instruments (also known as deflectable, rotatable, or articulated instruments) have been developed [5-9]. The advantage of these instruments compared with the conventional ones is that they provide sideways rotations of the tip.

Robotic manipulators, e.g., as in the Da Vinci Surgical Robot (Intuitive Surgical, Inc., Sunnyvale, CA) [10, 11], provide the surgeon with DOFs equal to those in conventional open surgery [12]. However, high costs, size, and lack of force feedback are the main factors for restricted use of those manipulators [10, 13, 14]. An affordable alternative to those robotic manipulators are hand-held mechanical manipulators $[5,8,12]$.

Most of the mechanical manipulators use wrist control to perform rotations of the instrument tip $[5,8,9,12]$. The 
Radius Surgical System (Radius) is an example of such a mechanical manipulator [5]. The wrist control also is used in the minimally invasive manipulator (MIM) developed by Jaspers et al. [8], as well as in the commercially available Autonomy Laparo-Angle instrument [9]. In the study by Gossot and Lange [7], the tip of the instrument is steered by a single control wheel.

To investigate whether using steerable instruments has an advantage compared with using conventional instruments, a few studies have been conducted. Martinec et al. [9] found that the conventional instruments slightly outperform the articulating autonomy Laparo-Angle instruments, taking time and accuracy during two suturing tasks into account. Conversely, Waseda et al. showed that using additional DOFs (as in the Radius) contributes to better accuracy and control of needle guiding compared with a conventional instrument, especially when difficult spatial situations occur [5]. The performance was assessed by means of time, accuracy, and number of actions. Focacci et al.[15] stated, however, that the controls of the Radius are not intuitive and a special training is required to achieve a good dexterity.

An optimal control of steerable instruments remains unsolved; there is lack of information about control mechanism that provides the surgeon with intuitive handling and better ergonomic performance. At the Department of BioMechanical Engineering, we have developed mechanisms for articulated instruments $[14,16]$. One of them-a spatial parallelogram mechanism-has been inspired by squid tentacles [16]. That mechanism allows deflection (hinge) of the instrument tip in two directions as well as axial tip rotation. The goal of this study was to compare easiness and intuitiveness of using interfaces to control steerable prototypes that make use of spatial parallelogram mechanism, as designed by Breedveld and Hirose [14]. Two controlling mechanisms: joystick ("thumb") and joint-articulation ("wrist") mechanisms were investigated. Both of those mechanisms had similar working principles and provided angular deflection of the control equal to that of the tip. The thumb/wrist control deflection remained parallel in this study in order to resemble original design of the instruments.

Because articulated instruments will be mostly used for complex surgical tasks, such as intracorporeal suturing or knot-tying, the control interfaces were examined using a needle-driving task performed in a box trainer. Two 5-mm prototypes were used in this study. Assessment of the prototypes was performed by means of motion analysis parameters (time included), accuracy, and a questionnaire. Additionally, the difficulty of performing the task in three different directions had been investigated. For that reason, a conventional instrument has been used besides the prototypes.

\section{Materials and methods}

The study was executed in the skills laboratory of Leiden University Medical Center (LUMC) in the Netherlands.

\section{Participants}

Right-handed medical students (novices, $n=12$ ) in their fourth to sixth academic year and right-handed laparoscopic surgeons (experts, $n=5$ ) participated in this study. The novices had no prior experience with laparoscopic surgery, whereas all experts had performed at least 100 laparoscopic procedures. All novices were asked to complete a short questionnaire that detailed demographic information and their prior experience in suturing.

\section{Prototypes}

Two 5-mm prototypes that make use of a spatial parallelogram mechanism [14] have been built by the DEAM corporation (www.deamcorporation.com) specifically for this study (Fig. 1). One of the prototypes was controlled by a thumb mechanism (Fig. 1B) and the second one by a wrist mechanism (Fig. 1C). Additionally, both prototypes consisted of a rotational wheel, which allowed rotation of the tip of the prototype around its axis. Both prototypes offered three DOFs at the tip: axial rotation, and the deflection of the tip in two directions. Angular deflection as provided by control mechanism was equal to that of the tip. A rotational wheel was not present in a conventional instrument, which was used to investigate the difficulty of the task (Fig. 1A).

\section{Task}

All participants were asked to perform a needle-driving task in a box trainer (Figs. 2, 3), with the three different instruments shown in Fig. 1. The task involved driving a 24-mm FS-1 curved needle through an artificial skin pad. Each participant proceeded the needle driving after an exact insertion of the needle in the predefined insertion point (Fig. 3). Additionally, he or she had to exit the tip of the needle as close to the predefined exit point as possible (Fig. 3). All of the participants performed the task in three directions: right $\rightarrow$ left $(\mathrm{R} \rightarrow \mathrm{L})$, up $\rightarrow$ down $(\mathrm{U} \rightarrow \mathrm{D})$, and down $\rightarrow$ up (D $\rightarrow$ U) (Fig. 3). For each direction, the "reference point" (indicating start/end position of the prototype or conventional instrument), "insertion point," and "exit point" were separately defined (Fig. 3).

For all participants, the position of the task and the placement of the camera in the box trainer were standardized. The workstation was adjusted ergonomically for each participant's height. All participants performed the 
Fig. 1 Three instruments used in this study. $U p$ a conventional needle holder. Middle a prototype of the instrument with the tip steered by a thumb mechanism. Bottom a prototype of the instrument with the tip steered by a wrist mechanism
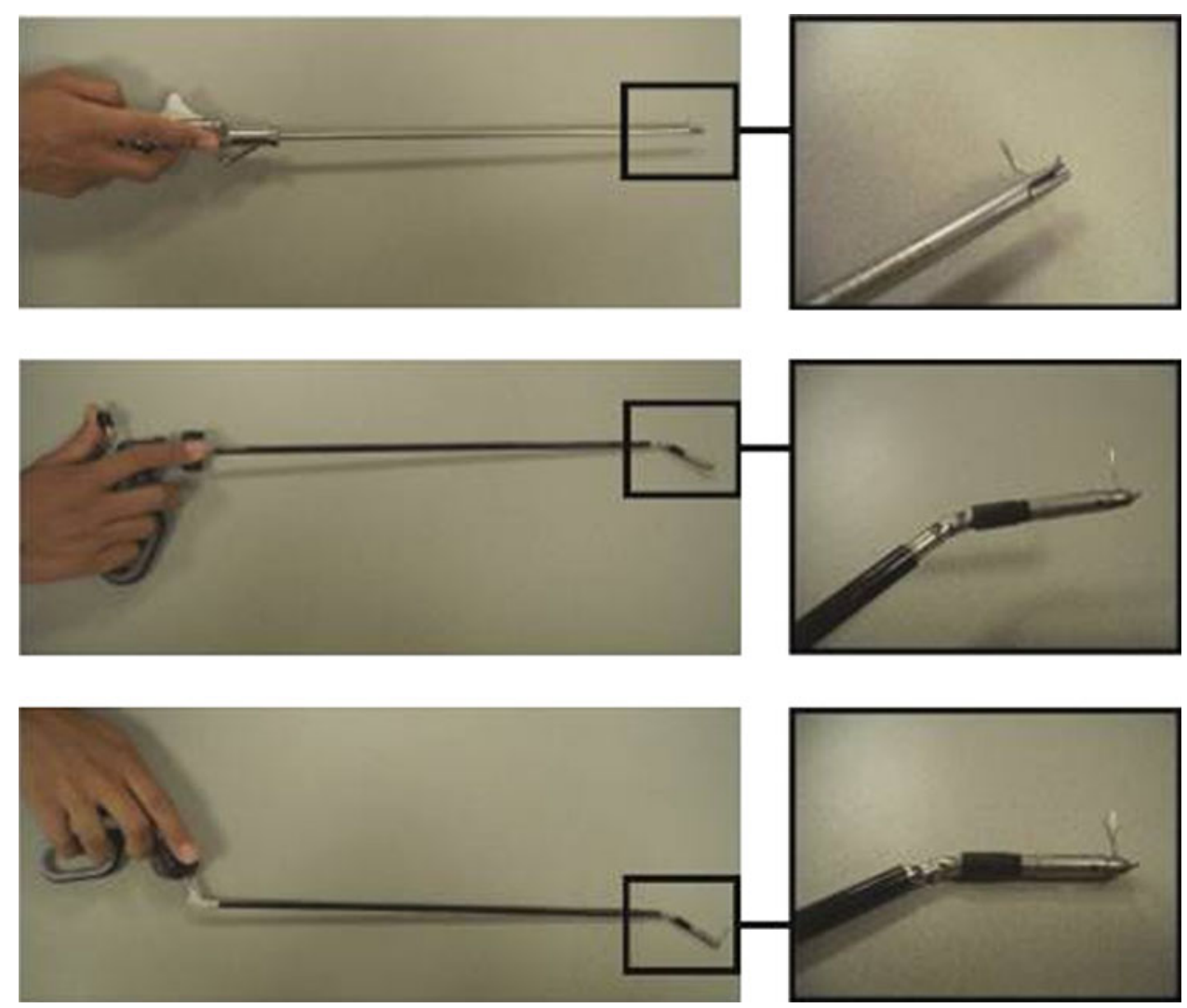

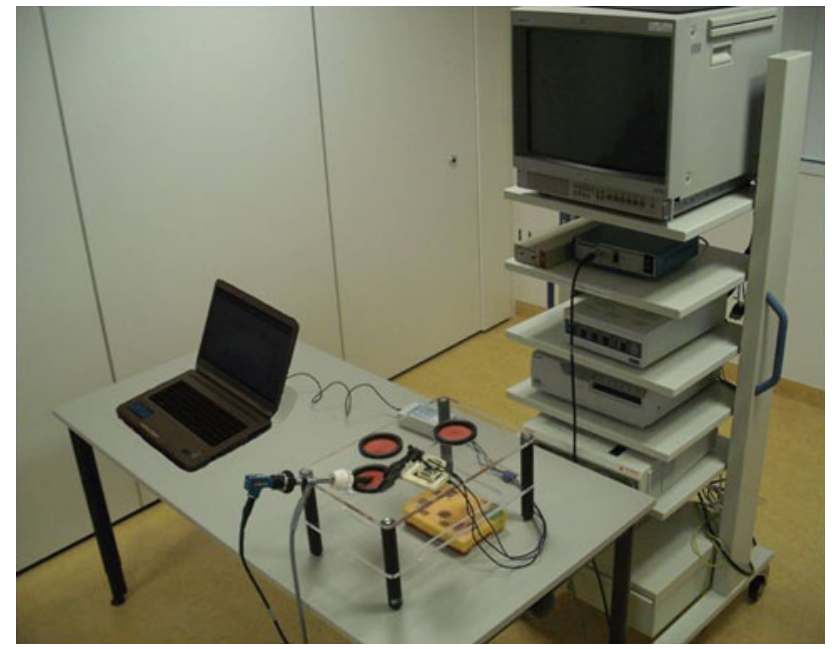

Fig. 2 Testing setup: a box trainer with a target plane (artificial skin pad), TrEndo tracking system, computer used to collect data, and the video system. The cover of the box trainer was nontransparent during performing the tasks

tasks with the dominant hand. Because no assistant instrument was used, the participants were allowed to keep their nondominant hand in any position comfortable for them. The camera was positioned on the left side of the participant and was held by an assistant. The assistant was always the same. The image of a $0^{\circ}$ laparoscope was presented on a monitor. Upon request, the assistant zoomed the view in or out.

\section{Experimental protocol}

The study was distributed over three sessions. Each consecutive session took place after at least 3 days. During every session, participants performed the task three times with the three instruments in each direction $(\mathrm{R} \rightarrow \mathrm{L}$, $\mathrm{U} \rightarrow \mathrm{D}$, and $\mathrm{D} \rightarrow \mathrm{U}$ ). In total, 27 tasks were performed during one session. During the three sessions, in total 81 tasks were performed. Each possible combination of instrument and task direction occurred nine times. On average, each session took $1.5 \mathrm{~h}$. The order of performing all tasks during the sessions was determined by combinations of Latin squares of task directions within Latin squares of instruments, to eliminate order of effects. The experts performed one session only, as they were familiar with laparoscopic suturing. The novices performed all three sessions.

The way the tasks needed to be performed was presented to the participants by means of a written manual with pictures and a verbal explanation. It was made clear that precision was given priority over time. Before the start of the experiment, the participants were permitted to practice with both prototypes and the conventional instrument on the artificial skin pad. This practice was allowed for 
maximal 5 min per instrument and was done outside the box trainer.

Outcome measures

The accuracy of the performed needle-driving tasks was objectively measured using a penalty matrix developed specially for this study (Fig. 3); the further the needle came out from the predefined exit point, the more penalty points were given.

The maximum time to perform each task was set at $10 \mathrm{~min}$. In case a participant was not able to perform the task in that time or if he gave up due to frustrations, for example, 12 penalty points were given.

Time taken to perform the task and movements of the instrument were recorded with a built-in TrEndo tracking system developed at Delft University of Technology (Fig. 2) [17]. The data were analyzed using five motion analysis parameters (MAPs):

1 Time - the total time taken to perform the task (in seconds).

2 Axial path length-the total distance travelled by the instrument along its axis (in millimeters).
Fig. 3 The needle-driving task and the penalty matrix. The insertion, exit, and reference points, together with the penalty matrices, are presented for the up $\rightarrow$ down direction (up), down $\rightarrow$ up direction (middle), and right $\rightarrow$ left direction (bottom). The number of penalty points that is assigned to a needle-driving task is determined by the square in the penalty matrix, where the needle exits the artificial skin pad. Within the square, one of four subpenalties is added, determined by the quadrant in the square from where the needle exits

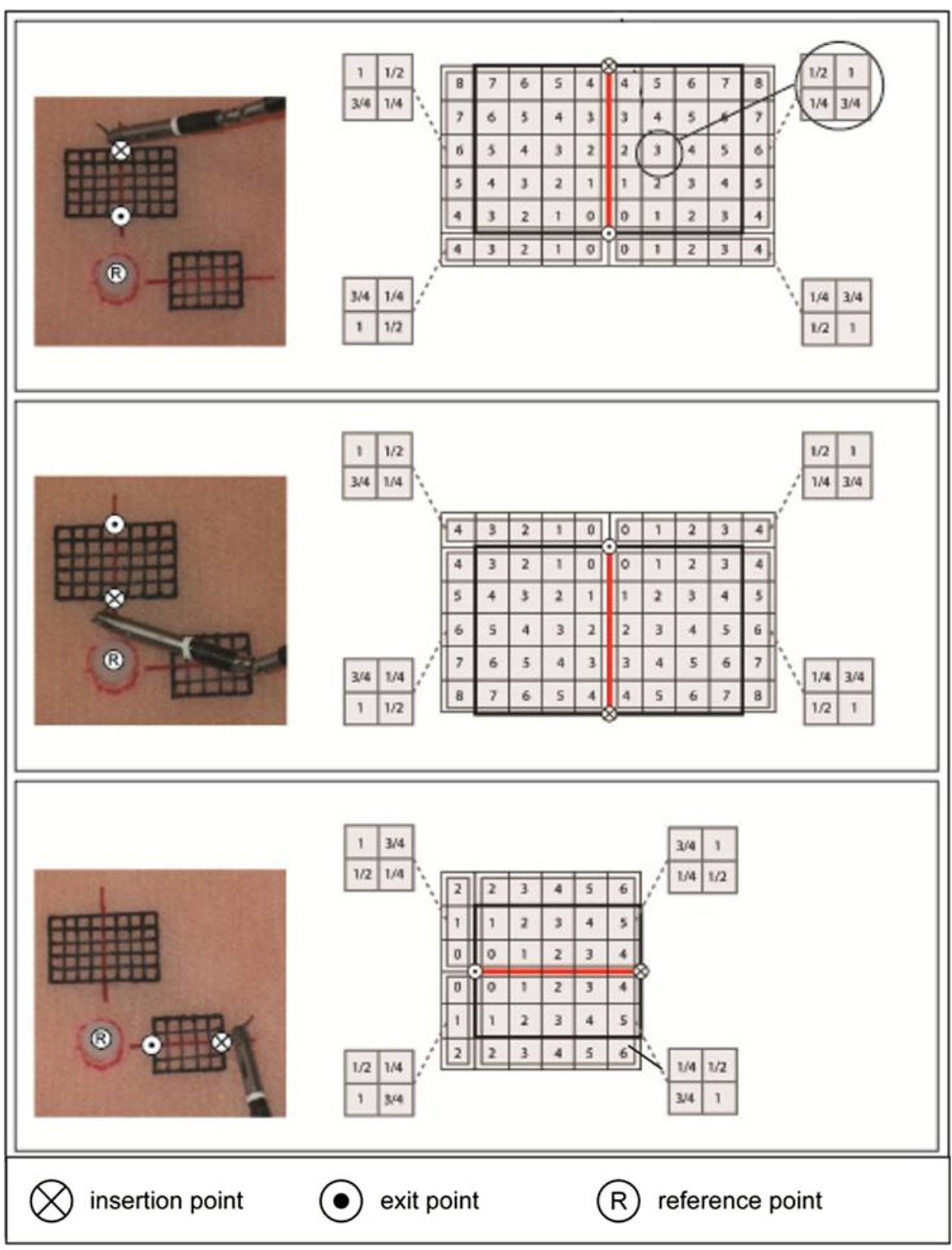


3 Rotational orientation - the amount of rotation of the instrument around its axis (in radians).

4 Rotational orientation alpha $(\alpha)$ - the amount of rotation of the instrument around the incision point in leftright direction (in radians).

5 Rotational orientation phi $(\varphi)$ - the amount of rotation of the instrument around the incision point in forwardbackward direction (in radians).

Because the design of the TrEndo allows measuring the movements at the incision point [17], it was impossible to track the movements of the tips of the prototypes. Therefore, in this study, axial path length, rotational orientation, $\alpha$, and $\varphi$ represent the changes at the incision point.

\section{Questionnaire}

After completing all three sessions, the novices were asked to fill out a questionnaire that comprised a general rating of each prototype and rewarding the following features of the prototypes:

1 Difficulty of performing a smooth movement to the insertion point.

2 Difficulty of positioning the needle at the insertion point.

3 Difficulty of driving the needle through the tissue in the required direction.

4 Discomfort during use of the prototype (e.g. cramp, pain in the muscle).

5 Difficulty of steering (controlling) the prototype.

6 Intuitiveness of steering the prototype.

7 Improvement of skills sensed after the last (third) session.

For this ranking, the novices were asked to give a score from 1 to 10 to each prototype for the above-mentioned features. The experts were asked to give their opinion about both prototypes.

Statistical analysis

Data were analyzed by using the SPSS 17.0 software package (SPSS, Chicago, IL). Statistical analyses were performed using repeated measures two-way analysis of variance (ANOVA) by applying mixed models. A Bonferroni-Holm modification was used to perform pairwise post-hoc analyses. $P$ values $<0.05$ were considered statistically significant.

\section{Results}

All 12 novices completed the entire study protocol. Due to time restrictions, three experts finished only a part of the session (20, 18, and 15 tasks, respectively), and two experts completed the whole session. Experts did not practice outside the box trainer before the experiment.

Because of technical problems, a number of measurements, including axial path length, rotational orientation, and $\varphi$ could not be recorded. Regarding the novices, data of 72 measurements were used for further analysis for each of the MAPs mentioned above. For the experts, data of eight measurements (for each of the above mentioned MAPs) were used for further analysis.

\section{Prototypes}

In case of novices, thumb prototype outperformed the wrist prototype for most of the measured parameters (Figs. 4, 5). No significant difference between the steerable prototypes was found in rotational orientation and axial path length (apart from the $\mathrm{D} \rightarrow \mathrm{U}$ task) as well as for the $\mathrm{R} \rightarrow \mathrm{L}$ task (apart from penalty points and rotational orientation alpha).

Statistical analysis was not performed for the experts due to a small amount of available data. Nevertheless, a trend was found showing that the better results are obtained with the thumb prototype than with the wrist prototype.

\section{Task}

The difficultness of the performed task in three directions was indicated by the penalty points. The results obtained by novices show that the difficultness of the $\mathrm{R} \rightarrow \mathrm{L}$, $\mathrm{U} \rightarrow \mathrm{D}$, and $\mathrm{D} \rightarrow \mathrm{U}$ task depended on the instrument used. In case of the thumb prototype, the best results were achieved for both the $\mathrm{R} \rightarrow \mathrm{L}$ and $\mathrm{D} \rightarrow \mathrm{U}$ tasks. For the wrist prototype, the best results were obtained for the $\mathrm{D} \rightarrow \mathrm{U}$ task. The results of the $\mathrm{R} \rightarrow \mathrm{L}$ task were better than those of the $\mathrm{U} \rightarrow \mathrm{D}$ task. In case of the conventional instrument, the best results were obtained for the $\mathrm{R} \rightarrow \mathrm{L}$ task. The results of the $\mathrm{D} \rightarrow \mathrm{U}$ task were better than those of the $\mathrm{U} \rightarrow \mathrm{D}$ task.

In case of experts, performing the task in all directions appeared to be comparable for the conventional instrument and both prototypes, except for the penalty points in which the $\mathrm{R} \rightarrow \mathrm{L}$ task and the $\mathrm{D} \rightarrow \mathrm{U}$ task showed better results than the $\mathrm{U} \rightarrow \mathrm{D}$ task (Fig. 6).

\section{Questionnaire}

The thumb prototype (with an average score of 7.0) scored higher than the wrist prototype (with an average score of 3.5). With respect to all the seven assessed features of the prototypes, the thumb prototype scored higher than the wrist prototype. 
Fig. 4 The results (penalty points, time, and axial path length) of the three tasks performed by the novices using the two interfaces to control steerable prototypes. Results are presented as box and whisker plots, where every box has a line at every quartile, median, and upper quartile values. The whiskers are presented as lines that extend from each end of the box to show the extent of the rest of the data; a few extreme outliers are excluded from the plots to omit too much compression of the $y$-axis. $* P<0.05$; ** $P<0.01$; $* * * P<0.001 ;$ present differences between mean values. $T$ thumb-steered prototype, $W$ wrist-steered prototype, $R \rightarrow L$ right $\rightarrow$ left task, $U \rightarrow D$ up $\rightarrow$ down task, $D \rightarrow U$ down $\rightarrow$ up task
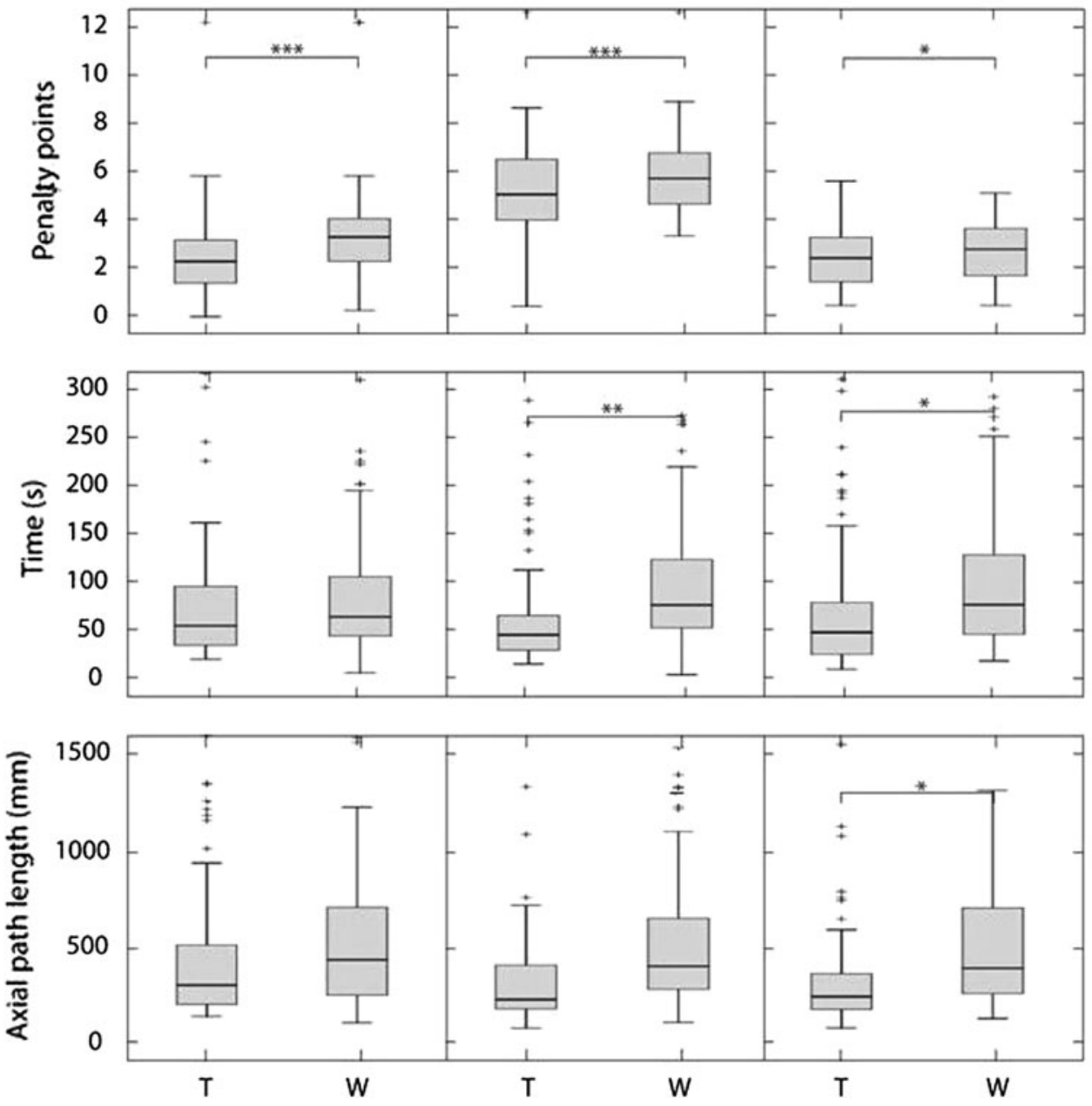

\section{Discussion}

This study shows that the joystick (thumb) interface to control steerable prototypes does have an advantage over a joint-articulation (wrist) interface, with respect to motion analysis parameters, accuracy, and participants' subjective perception of the prototypes. This has been found for both the experts and the novices. Nearly all participants stated that they had problems with controlling the wrist prototype due to the movable shaft, which made the prototype "too flexible" at the moments when it was preferable to have a more rigid prototype. In consequence, the participants made more uncontrolled movements and had overall worse performances, which were reflected in motion analysis parameters and accuracy measures.

In both prototypes, it was impossible to choose a correct angle of the tip (sideways rotation) and to lock it such that the prototype would remain rigid at that particular configuration. In opposite to the wrist prototype, the thumb prototype consisted of a rigid shaft. It was, therefore, possible to use the thumb prototype identically as a conventional instrument. Some of the participants (especially the experts) made use of that; they did not apply additional forces with their thumb (to keep the tip of the instrument at the constant angle). As a result, those participants performed the task better than those who applied forces with the thumb. It was observed that participants tended to get used to the thumb interface very fast, because it was much easier for them to keep the prototype at the desired position while orienting its tip. Moreover, almost all participants admitted that adding a "locking" feature would result in an easier way of controlling both prototypes, especially when applying forces to the tissue.

The study by Zahraee et al. [13] in the virtual environment without force feedback showed that joystick interface is preferable and easier to use compared with a jointed interface. Furthermore, Zahraee et al. [18] showed that the locking feature helps the surgeon to perform more precise gestures. Our data indicate that the locking feature is necessary in steerable instruments. In our tests environment, the application of pushing forces was required. We found that without the locking feature, keeping the tip of the prototype at the constant angle while applying forces to the tissue is difficult and can result in uncontrolled 
Fig. 5 The results (rotational orientation, rotational orientation alpha, and rotational orientation phi) of the three tasks performed by the novices using the two interfaces to control steerable prototypes. $* P<0.05 ; * * P<0.01$; $* * * P<0.001 ;$ present differences between mean values. $T$ thumb-steered prototype, $W$ wrist-steered prototype, $R \rightarrow L$ right $\rightarrow$ left task, $U \rightarrow D$ up $\rightarrow$ down task, $D \rightarrow U$ down $\rightarrow$ up task
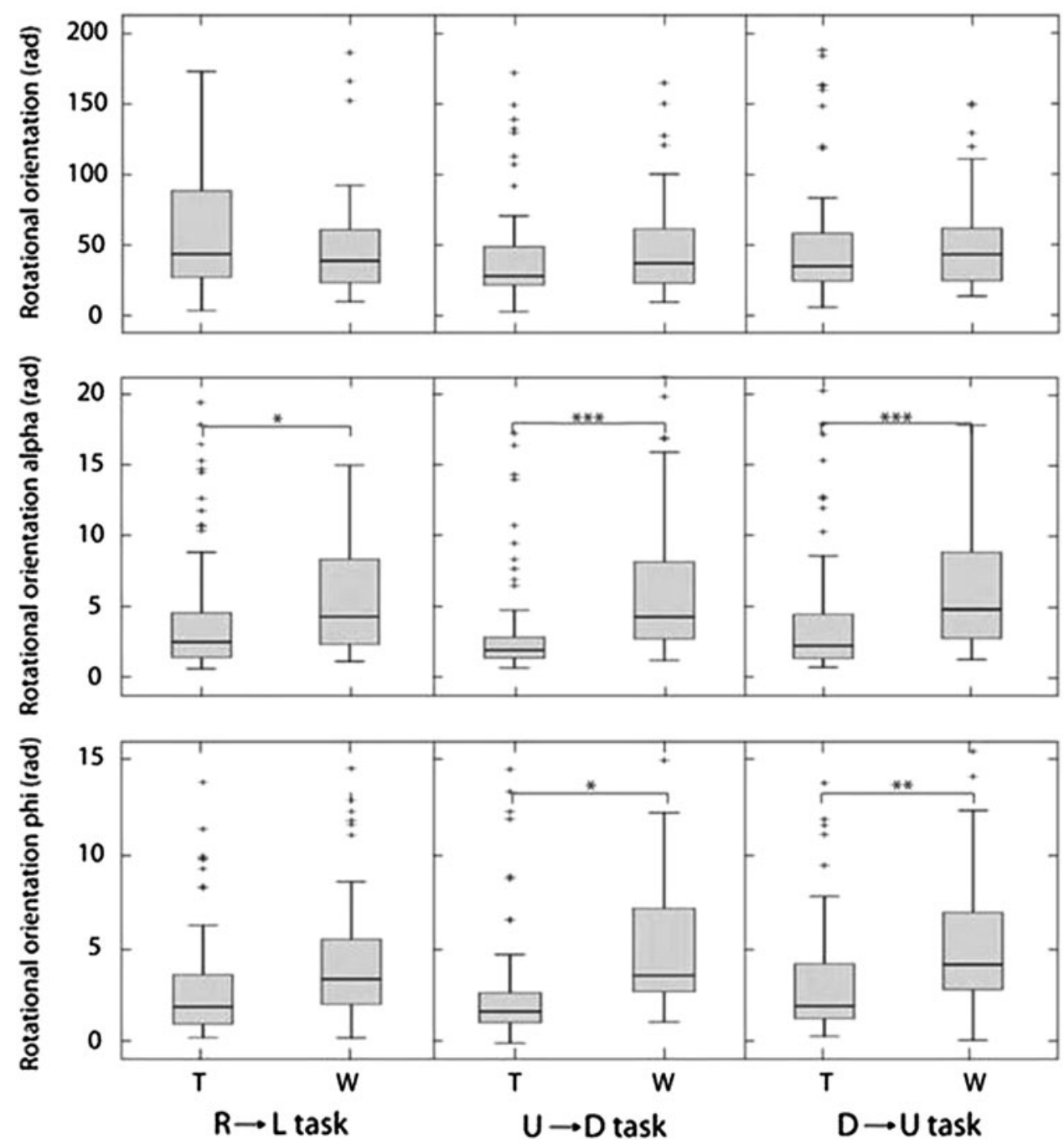

Task

R-L

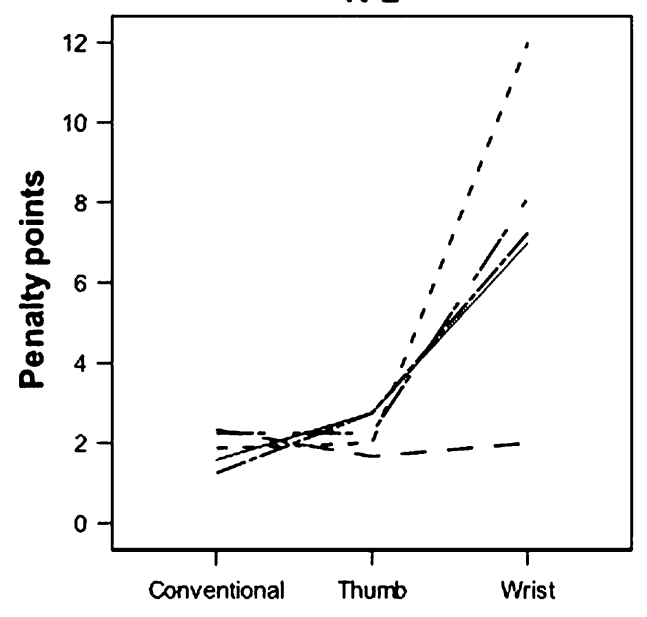

U-D

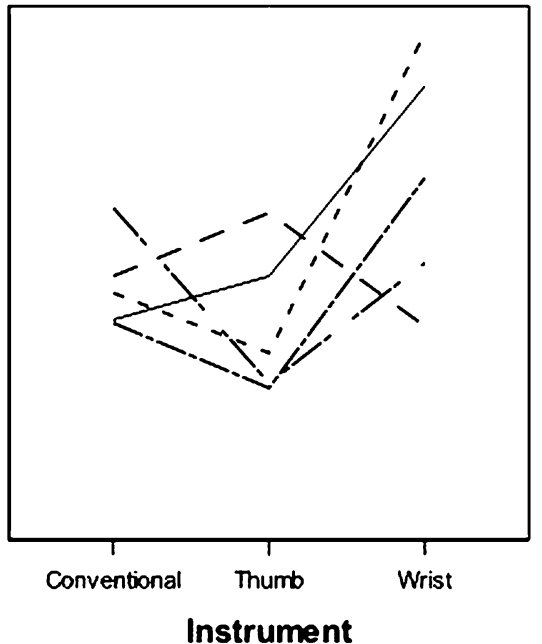

D-U

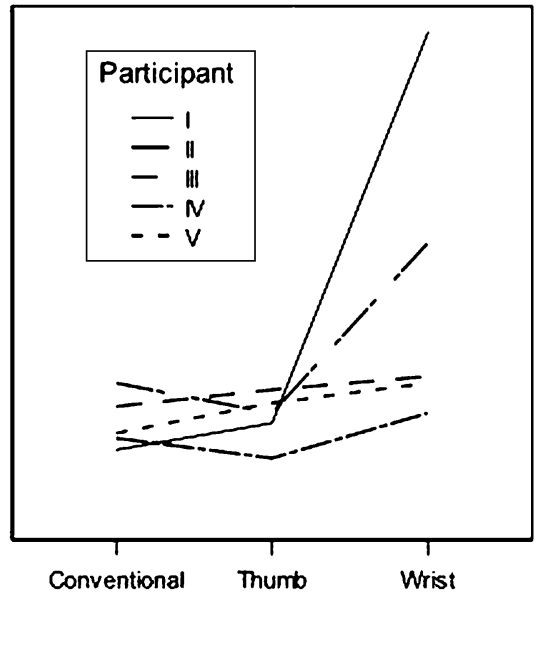

Fig. 6 The results of the three tasks performed by the experts using the conventional instrument and the two interfaces to control steerable prototypes. Results are presented as lines, in which each line represents an experts' mean score of penalty points 
movements. This shows that the presence of force feedback influences basic laparoscopic skills during tasks in which pulling and pushing forces are applied [19, 20]. Therefore, evaluations of laparoscopic instruments and techniques should be performed in environments in which force feedback is present.

Currently, articulated instruments are being used by experienced surgeons to perform difficult tasks and procedures (e.g., suturing, single-port surgery). We believe that novices also could benefit from using articulated instruments. For example, an articulated endoscope might be used to obtain a natural line-of-sight, which should improve novice's eye-hand coordination. Therefore, the easiness and intuitiveness of using both prototypes in this study has been investigated by analyzing performance of the novices. This has an advantage of not biasing the outcome; the novices did not have any experience with laparoscopic instruments and, by consequence, their performance was not influenced by familiarity with such instruments. The performance and comments of both novices and experts will be used in further development of the articulated instruments that make use of a spatial parallelogram mechanism.

Our results show that the conventional instrument performed well and, in some cases, it outperformed prototypes. This is an important finding, which deserves attention. We think that articulated instruments can be useful and, if they are carefully and ergonomically designed, they can help to perform complicated tasks. Our study shows that if care is not taken in the design of the articulated instruments (e.g., when locking feature is not included), using those instruments might cause problems. Therefore, ergonomic evaluation of the articulated instruments is of great importance. The main focus of this study was comparison of two prototypes of articulated instruments. Because those prototypes are still in the development stage (they are not "end products" yet), it is difficult to make an honest comparison of them and conventional instrument.

To our knowledge, this is one of the first studies to compare control methods of articulated instruments by means of motion analysis [22]. Other studies have shown that motion analysis can be used to objectively assess psychomotor laparoscopic skills [21-30]. Besides that, it has been shown that motion analysis can be used to assess difficulty of performed task [31]. With this study, we confirm the finding of Kolwadkar et al. [22] that motion analysis can be used to investigate which instruments are suitable to perform particular laparoscopic tasks.

The needle-driving task used in this study is a part of a suturing task. To mimic an in vivo situation, the task was performed in three directions: $\mathrm{R} \rightarrow \mathrm{L}, \mathrm{U} \rightarrow \mathrm{D}$, and $\mathrm{D} \rightarrow \mathrm{U}$. The $\mathrm{L} \rightarrow \mathrm{R}$ direction was excluded, because it required an unnatural way of performing the task. (The needle-driven task was placed in the middle of the box trainer and was approached by the instrument from the right side.) We have found that for both novices and experts, the up-down task was the most difficult to perform with the conventional instrument as well as with the prototypes. It is, therefore, advisable not to perform suturing in this direction.

The main focus of this study was on assessment of joystick and wrist control in hand-held articulated instruments. Further studies should investigate when (what kind of tasks and procedures) it could be advantageous to use articulated instruments instead of the conventional ones. Additionally, measures, such as physical workload, should be included in those studies to obtain supplementary information on advantages and disadvantages of using articulated instruments.

\section{Conclusions}

The thumb interface to control steerable prototype outperformed the wrist interface, with respect to motion analysis parameters, accuracy, and participant's subjective perception of the prototypes. To avoid uncontrolled movements and difficulties with applying forces to the tissue, while keeping the tip of the instrument at the constant angle, adding a locking feature is necessary. It is advisable not to perform the needle driving task in the up to down direction.

Acknowledgments The authors thank the DEAM Corporation for developing the prototypes used in this study and Ron Wolterbeek, MD, from the Leiden University Medical Center (LUMC), for useful comments during preparation of this paper. The authors also thank all surgeons and medical students for participating in this study and staff of the skills laboratory of LUMC for providing the available working space.

Disclosures Linde M. Okken, Magdalena K. Chmarra, Ellen Hiemstra, Frank-Willem Jansen, and Jenny Dankelman have no conflicts of interests or financial ties to disclose.

Open Access This article is distributed under the terms of the Creative Commons Attribution Noncommercial License which permits any noncommercial use, distribution, and reproduction in any medium, provided the original author(s) and source are credited.

\section{References}

1. Dankelman J, Grimbergen CA, Stassen HG (2005) Engineering for patient safety (issues in minimally invasive procedures). Lawrence Erlbaum, London

2. Cuschieri A (2005) Laparoscopic surgery: current status, issues and future developments. Surgeon 3:125-130, 132-133, 135-138 
3. Frede T, Stock C, Rassweiler MD, Alken P (2000) Retroperitoneoscopic and laparoscopic suturing: tips and strategies for improving efficiency. J Endourol 19:230-238

4. Emam TA, Hanna G, Cuschieri A (2002) Ergonomic principles of task alignment, visual display, and direction of execution of laparoscopic bowel suturing. Surg Endosc 16:267-271

5. Waseda M, Inaki N, Torres Bermudez JR, Manukyan G, Gacek IA, Schurr MO, Braun M, Buess GF (2007) Precision in stitches: radius surgical system. Surg Endosc 21:2056-2062

6. Melzer A, Kipfmuller K, Halfar B (1997) Deflectable endoscopic instrument system DENIS. Surg Endosc 11:1045-1051

7. Gossot D, Lange G (2001) Deflectable and rotatable endoscopic instrument with intuitive control. Minim Invasive Ther Allied Technol 10:295-303

8. Jaspers JEN, Bentala M, Herder JL, de Mol BA, Grimbergen CA (2004) Mechanical manipulator for intuitive control of endoscopic instruments with seven degrees of freedom. Minim Invasive Ther Allied Technol 13:191-198

9. Martinec DV, Gatta P, Zheng B, Denk PM, Swanström LL (2009) The trade-off between flexibility and maneuverability: task performance with articulating laparoscopic instruments. Surg Endosc 23:2697-2701

10. Ahmed K, Shamim Khan M, Vats A, Nagpal K, Priest O, Patel V, Vecht JA, Ashrafian H, Yang GZ, Athanasiou T, Darzi A (2009) Current status of robotic assisted pelvic surgery and future developments. Int J Surg 7:431-440

11. Bann S, Khan M, Hernandez J, Munz Y, Moorthy K, Datta V, Rockall T, Darzi A (2003) Robotics in surgery. J Am Coll Surg 196:784-795

12. Schurr MO, Buess G, Schwarz K (2001) Robotics in endoscopic surgery: can mechanical manipulators provide a more simple solution for the problem of limited degrees of freedom? Minim Invasive Ther Allied Technol 10:289-293

13. Zahraee AH, Szewczyk J, Morel G (2009) Simulation for optimal design of hand-held surgical robots. In: 31 st annual international conference of the IEEE EMBS, Minneapolis, 2-6 Sep 2009

14. Breedveld P, Hirose S (2001) Development of the endo-periscope. Minim Invasive Ther Allied Technol 10:315-322

15. Focacci F, Piccigallo M, Tonet O, Megali G, Pietrabissa A, Dario P (2007) Lightweight hand-held robot for laparoscopic surgery. In: IEEE international conference on robotics and automation, Roma, 10-14 Apr 2007

16. Breedveld P, Scheltes JS, Blom EM, Verheij JE (2005) A new, easily miniaturized steerable endoscope. Squid tentacles provide inspiration for the endo-periscope. IEEE Eng Med Biol Mag 24(6):40-47

17. Chmarra MK, Bakker NH, Grimbergen CA, Dankelman J (2006) TrEndo, a device for tracking minimally invasive surgical instruments in training setups. Sensor Actuat A 126:328-334
18. Zahraee AH, Szewczyk J, Morel G (2009) Evaluating control models for hand-held robotic surgical instrument using virtual reality simulator. In: IEEE/ASME international conference on advanced intelligent mechatronics, Singapore, 14-17 July 2009

19. Chmarra MK, Dankelman J, van den Dobbelsteen JJ, Jansen FW (2008) Force feedback and basic laparoscopic skills. Surg Endosc 22:2140-2148

20. Hiemstra E, Kolkman W, van de Put MAJ, Jansen FW (2009) Retention of basic laparoscopic skills after a structured training program. Gynecol Surg 6:229-235

21. Cotin S, Stylopoulos N, Ottensmeyer M, Neumann P, Rattner D, Dawson S (2002) Metrics for laparoscopic skill trainers: the weakest link! In: Lecture notes in computer science, vol 2488. Springer, New York, pp 35-43

22. Kolwadkar YV, Brown SI, Abboud RJ, Wang W (2011) Comparison of two actuation systems for laparoscopic surgical manipulators using motion analysis. Surg Endosc 3:964-974

23. Van Sickle KR, McClusky DA, Gallagher AG, Smith CD (2005) Construct validation of the ProMIS simulator using a novel laparoscopic suturing task. Surg Endosc 19:1227-1231

24. Acosta E, Temkin B (2005) Haptic laparoscopic skills trainer with practical user evaluation metrics. Stud Health Techn Inform MMVR 111:8-11

25. Cavallo F, Megali G, Sinigaglia S, Tonet O, Dario P (2005) A biomechanical analysis of surgeon's gesture in a laparoscopic virtual scenario. Stud Health Technol Inform MMVR 14:79-84

26. Chmarra MK, Klein S, De Winter JCF, Jansen FW, Dankelman J (2010) Objective classification of residents based on their psychomotor laparoscopic skills. Surg Endosc 24:1031-1039

27. Cesanek P, Uchal M, Uranues S, Patruno J, Gogal C, Kimmel S, Bergamaschi R (2008) Do hybrid simulator-generated metrics correlate with content-valid outcome measures? Surg Endosc 22:2178-2183

28. Allen B, Nistor V, Dutson E, Carman G, Lewis C, Faloutsos P (2009) Support vector machines improve the accuracy of evaluation for the performance of laparoscopic training tasks. Surg Endosc 24:170-178

29. Leong JJH, Nicolaou M, Atallah L, Mylonas GP, Darzi AW, Yang GZ (2006) HMM assessment of quality of movement trajectory in laparoscopic surgery. In: MICCAI, Lecture notes in computer science, vol 4190. Springer, New York, pp 752-759

30. Rosen J, Solazzo M, Hannaford B, Sinanan M (2001) Objective laparoscopic skills assessments of surgical residents using hidden Markov models based on haptic information and tool/tissue interactions. Stud Health Tech Inform MMVR 81:417-423

31. Chmarra MK, Kolkman W, Jansen FW, Grimbergen CA, Dankelman J (2007) The influence of experience and camera holding on laparoscopic instrument movements measured with the TrEndo tracking system. Surg Endosc 21:2069-2075 